\title{
Critical exponents in stochastic sandpile models
}

\author{
Alessandro Chessa ${ }^{1}$, Alessandro Vespignani ${ }^{2}$ \\ and Stefano Zapperi ${ }^{3}$ \\ ${ }^{1}$ Dipartimento di Fisica and Unitá INFM, Universitá di Cagliari, \\ Via Ospedale 72, 09124 Cagliari, Italy \\ ${ }^{2}$ The Abdus Salam International Centre for Theoretical Physics (ICTP), \\ P.O. Box 586, 34100 Trieste, Italy \\ ${ }^{3}$ PMMH-ESPCI, 10 Rue Vauquelin, 75234 Paris CEDEX 05, France
}

\begin{abstract}
We present large scale simulations of a stochastic sandpile model in two dimensions. We use moments analysis to evaluate critical exponents and finite size scaling method to consistently test the obtained results. The general picture resulting from our analysis allows us to characterize the large scale behavior of the present model with great accuracy.
\end{abstract}

Sandpile automata [1] are prototypical models to describe avalanche transport processes. All these models show a stationary state that after a suitable tuning of the driving fields[2] displays a singular response function characterized by power law distributed events. These distributions are typically bounded by upper cut-offs related to the system size. In analogy with critical phenomena, is possible to define a complete set of scaling exponents describing the large scale behavior of these models.

Despite the large conceptual impact and the huge effort devoted to the study of sandpile automata in the last ten years, many basic issues, such as the precise values of the critical exponents, the identification of universality classes and of the upper critical dimension, still lay unresolved. Theoretically, many approaches [3, 4, 5] point out that different sandpile models, such as the Bak, Tang and Wiesenfeld (BTW) [1] and the Manna [6] models, all belong to the same universality class. Theoretical estimates for critical exponents have been provided (especially in Euclidean dimension $d=2$ ) by means of different methods [3, 4, 7], and some exact results [8] can be derived from the Abelian structure of the BTW model. Numerical results 
are difficult to interpret, since different methods of analysis typically yield different results [6, 9, 10, 11, 12, 13. This is probably due to intrinsic scaling anomalies and finite size effects present in sandpile models.

Here, we present very large scale numerical simulations of the Manna model [6], that is the standard example of a sandpile automaton with stochastic toppling rule. We show that Manna model can be coherently described within a finite size scaling (FSS) framework. Critical exponents are evaluated with great accuracy and the results are confirmed by data collapse analysis.

We consider a two-dimensional square lattice of linear size $L$ and associate to each site an integer variable $z_{i}$ (energy). At each time step an energy grain is added on a randomly chosen site $\left(z_{i} \rightarrow z_{i}+1\right)$. When one of the sites reaches or exceeds the local threshold $z_{c}=2$ a "toppling" occurs:: $z_{i}=z_{i}-2$ and $z_{j}=z_{j}+1$, where $j$ represents two randomly chosen nearest neighbor sites of site $i$. A toppling can induce nearest-neighbor sites to topple on their turn and so on, until all the lattice sites are below the critical threshold. This process is called an avalanche. Grains are added only when all the sites are below the threshold, which corresponds to a fine tuning of the external driving field [2]. In addition, the model is conservative and energy is dissipated only at boundary sites [1, [].

Avalanches in sandpile models are usually characterized by three variables: the number of topplings $s$, the area $a$ affected by the avalanche, and the avalanche duration $t$. The probability distribution of each of these variables can be described as a power law with a cutoff

$$
P(x)=x^{-\tau_{x}} \mathcal{G}\left(x / x_{c}\right),
$$

where $x=s, a, t$. When the system size $L$ goes to infinity the cutoff $x_{c}$ diverges as $x_{c} \sim L^{\beta_{x}}$. Under the finite size scaling (FSS) assumption of Eq. (11), the set of exponents $\left\{\tau_{x}, \beta_{x}\right\}$ defines the universality class of the model. In order to test the above FSS picture and to find an accurate estimate of the various critical exponents, we perform numerical simulations of two-dimensional Manna model with open boundary conditions and conservative dynamics. The lattice size ranges from $L=128$ to $L=2048$, and statistical distributions are obtained averaging over $10^{7}$ nonzero avalanches. The direct numerical determination of the exponents $\tau_{x}$ from the power law behavior of the probability distributions contains intrinsic bias due to the lower and upper cut-offs. This makes very difficult to get better than a 10\% accuracy. Extrapolations methods have been devised [13], but the estimate of their accuracy is rather difficult. For these reasons, we use the 

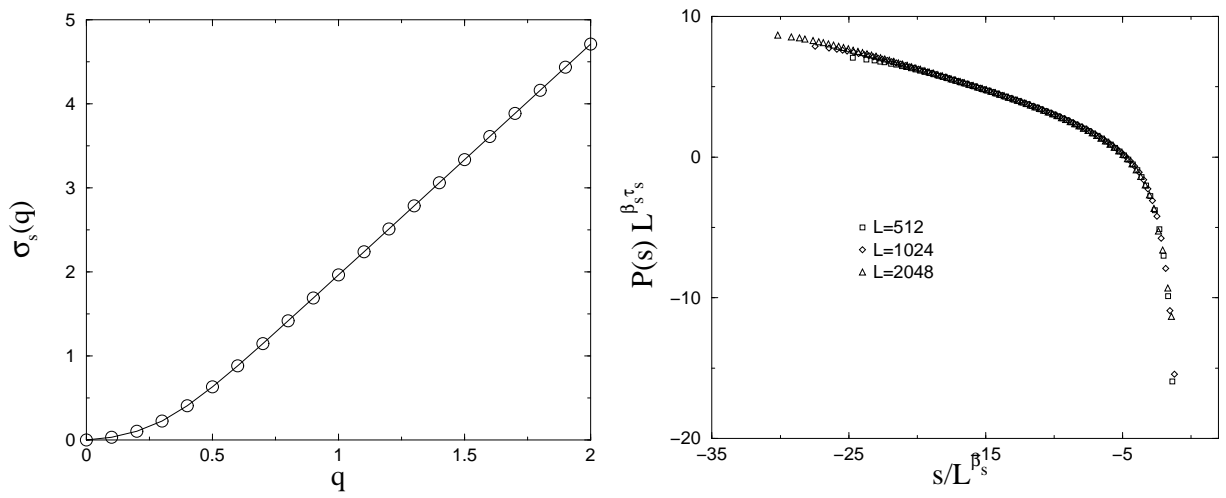

Figure 1: (a) Plot of the moments spectrum for the distribution of toppling events $s$.The linear part has slope 2.73.(b) Data collapse analysis for the avalanche size distribution. The values used for the critical exponents are $\tau_{s}=1.27$ and $\beta_{s}=2.7$.

more powerful moments analysis on the distribution $P(x, L)$, as also suggested recently by De Menech et al.[11] on the BTW model. We define the $q$-moment of $x$ on a lattice of size $L$ as $\left\langle x^{q}\right\rangle_{L}=\int x^{q} P(x) d x$. If FSS hypothesis (Eq. (11)) is valid, at least in the asymptotic limit $(x \rightarrow \infty)$, we can transform $z=x / L^{\beta_{x}}$ and obtain

$$
\left\langle x^{q}\right\rangle_{L}=L^{\beta_{x}\left(q+1-\tau_{x}\right)} \int z^{q+\tau_{x}} \mathcal{G}(z) d z \sim L^{\beta_{x}\left(q+1-\tau_{x}\right)},
$$

or in general $\left\langle x^{q}\right\rangle_{L} \sim L^{\sigma_{x}(q)}$. The exponents $\sigma_{x}(q)$ can be obtained as the slope of the log-log plot of $\left\langle x^{q}\right\rangle_{L}$ versus $L$. Using Eq. (2), we obtain $\left\langle x^{q+1}\right\rangle_{L} /\left\langle x^{q}\right\rangle_{L} \sim L^{\beta_{x}}$ or $\sigma_{x}(q+1)-\sigma_{x}(q)=\beta_{x}$, so that the slope of $\sigma_{x}(q)$ as a function of $q$ is the cutoff exponent $\beta_{x}=\partial \sigma_{x}(q) / \partial q$. In Fig.s 1(a),2(a) and $3(\mathrm{a})$, we show the result obtained from the moments analysis of the distribution $P(s), P(t)$ and $P(a)$, respectively. In all cases, we get a clear linear behavior starting from $q \simeq 0.7$. For smaller $q$ we observe deviations from standard FSS, expected because the integral in Eq. (2) is dominated by the lower cutoff for small $q$ moments. In particular, corrections to scaling of the type $\left\langle x^{q}\right\rangle_{L} \sim L^{\sigma_{x}(q)} F(L)$ are important for $q \leq \tau_{x}-1$ and when $q \simeq$ $\tau_{x}-1$, logarithmic corrections give rise to effective exponents up to very large lattice sizes. By measuring the slope of the linear part of momentum spectra $\sigma_{x}(q)$, we obtain the cut-offs exponents $\beta_{s}=2.73 \pm 0.02, \beta_{t}=1.50 \pm 0.02$ and $\beta_{a}=2.02 \pm 0.02$. These exponents 14 are in good agreement with previous estimates for the manna model [6]. If FSS is verified, we can compute the 

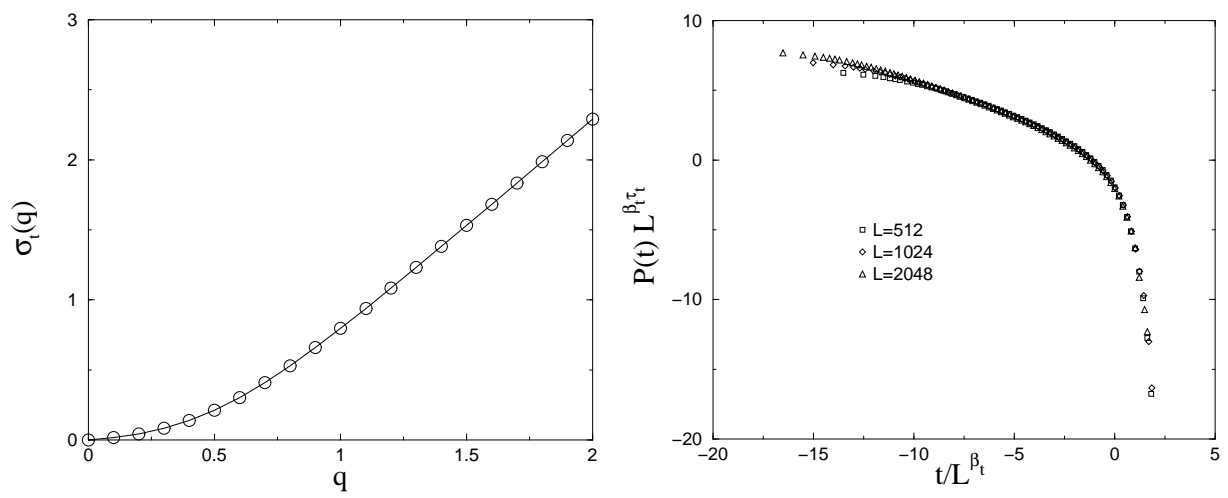

Figure 2: (a) Plot of the moments spectrum for the distribution of avalanche time duration $t$.The linear part has slope 1.50.(b) Data collapse analysis for the avalanche time duration distribution. The values used for the critical exponents are $\tau_{t}=1.5$ and $\beta_{a}=1.5$.

exponent $\tau_{x}$ from the scaling relation $\left(2-\tau_{x}\right) \beta_{x}=\sigma_{x}(1)$, that should be satisfied for enough large sizes. Using the values of $\beta_{x}$ reported in Table I and the values obtained for $\sigma_{x}(1)$ we find the exponents $\tau_{s}=1.27 \pm 0.01$, $\tau_{t}=1.50 \pm 0.01$ and $\tau_{a}=1.35 \pm 0.01$. Also in this case the values are in agreement with previous extrapolations or direct measurements [6, 13]. As a final consistency test for the FSS framework we have to verify that we get data collapse for the distributions $P(x . L)$ by using the exponents obtained from the moments analysis. In fact, the FSS scenario states that rescaling $q_{x} \equiv x / L^{\beta_{x}}$ and $P_{q_{x}} \equiv P(x, L) L^{\beta_{x} \tau_{x}}$, the data for different $L$ must collapse onto universal curves. In Fig.s 1(b), 2(b) and 3(b), we show that very good data collapses are obtained for all distributions.

In conclusion, we have reported extensive numerical simulations of the Manna sandpile model in two dimensions [6]. We show that contrary to other sandpile models, such as the BTW model [1], where it is difficult to obtain unambiguous results 11, 13, the FSS assumption is satisfied in the Manna model. In this way, we obtain the complete set of avalanche critical exponents. Work is in progress to obtain comparable results in $d=3,4$.

\section{References}

[1] P. Bak, C. Tang and K. Wiesenfeld, Phys. Rev. Lett. 59, 381 (1987); Phys. Rev. A 38, 364 (1988). 

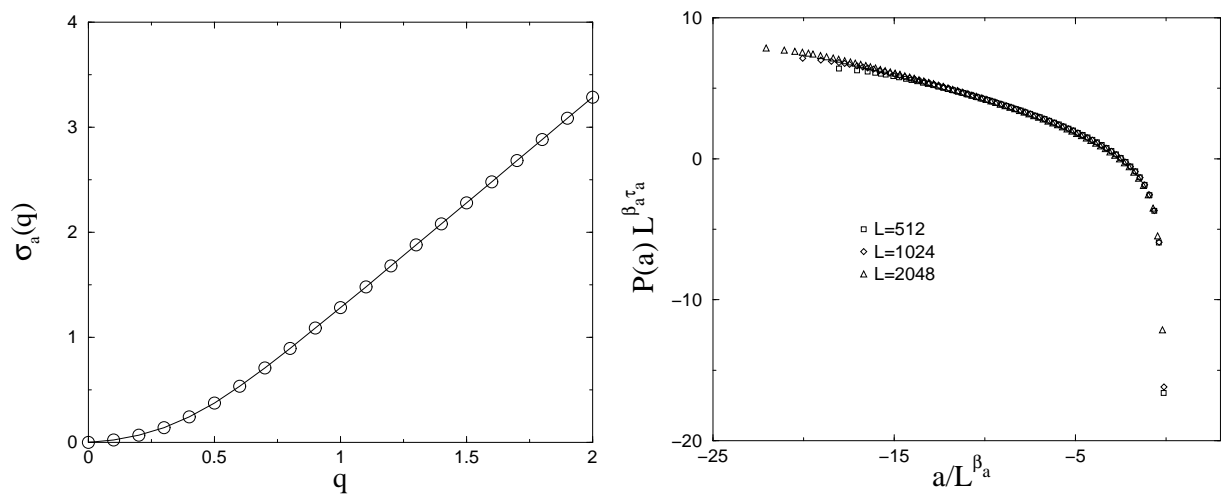

Figure 3: (a) Plot of the moments spectrum for the distribution of avalanche area $a$.The linear part has slope 2.02.(b) Data collapse analysis for the avalanche area distribution. The values used for the critical exponents are $\tau_{a}=1.35$ and $\beta_{a}=2.0$.

[2] A. Vespignani and S. Zapperi, Phys. Rev. Lett. 78, 4793 (1997); Phys. Rev. E 57, 6345 (1998).

[3] L. Pietronero, A. Vespignani and S. Zapperi, Phys. Rev. Lett. 72, 1690 (1994); A. Vespignani, S. Zapperi and L. Pietronero, Phys. Rev. E 51, 1711 (1995).

[4] A. Díaz-Guilera, Europhys. Lett. 26, 177 (1994); A. Corral and A. Díaz-Guilera, Phys. Rev. E 55, 2434 (1997).

[5] R. Dickman, A. Vespignani and S. Zapperi, Phys. Rev. E 57, 5095 (1998);A. Vespignani, R. Dickman, M. A. Muñoz, and S. Zapperi, preprint cond-mat/9806249.

[6] S. S. Manna, J. Phys. A 24, L363 (1991).

[7] V. B. Priezzhev, D. V. Ktitarev and E. V. Ivashkevich, Phys. Rev. Lett. 76, 2093 (1996).

[8] For a review see: D. Dhar, cond-mat/9808047.

[9] P. Grassberger and S. S. Manna, J. Phys. (France) 51, 1077 (1990); S. S. Manna, J. Stat. Phys. 59, 509 (1990); Physica A 179, 249 (1991).

[10] A. Ben-Hur and O.Biham, Phys. Rev. E 53, R1317 (1996); E. Milshtein, O. Biham and S. Solomon, Phys. Rev. E 58, 303 (1998). 
[11] M. De Menech, A. L. Stella and C. Tebaldi, Phys. Rev. E 58, R2677 (1998).

[12] A. Chessa, E. Marinari, A. Vespignani and S. Zapperi, Phys. Rev. E 57, R6241 (1998).

[13] S. Lübeck and K.D. Usadel, Phys. Rev. E 55, 4095 (1997); ibid. 56, 5138 (1997); S. Lübeck, ibid 56, 1590 (1997).

[14] Note that the exponents $\beta_{s}, \beta_{t}$ and $\beta_{a}$ are usually reported in the literature as $D, z$ and $d_{f}$, respectively. 